\title{
On the three laws of rotationally supported galaxies: the observed flattening of rotation curves, baryonic Tully-Fischer relation and the mass discrepancy-acceleration relation.
}

\author{
José Alcauza \\ jose.alcauzaatencia@telefonica.com
}

October, 2020

\begin{abstract}
In this paper we will find that, according to holographic principle [1] and thus considering Universe as the ensemble of $\aleph$ information bits or minimum particles of mass $m_{g}$, the contribution to galactic rotation curves can be due the rest of the visible Universe through a non-local collective gravitational interaction of all particles within the Universe's horizon, as a consequence of which all particles are gravitationally entangled and form a unified statistical ensemble. Therefore, we can to describe this global effect in terms of standard local Newtonian gravity within galaxies for the explanation of flatness galactic rotation curves as a possible alternative to the dark matter or MOND hypothesis.

We will find a solution for the baryonic Tully-Fischer relation: $M_{b}=A v_{f}^{4} \Longleftrightarrow A=\left[a_{0} G\right]^{-1}$ with $a_{0}=\frac{c H_{0}}{2 \pi}$, where $H_{0}$ is the Hubble constant at present Time $t_{0}$ and $M_{b}, G$ and $c$ are the galaxy baryonic mass, gravitational constant and constant speed of light in vacuum respectively. Also we will find the mass discrepancy-acceleration relation, thus obtaining a possible solution for each of the three laws of rotationally supported galaxies proposed in ([2],[3]).
\end{abstract}

Keywords: holographic principle; dark matter; MOND; flatness rotation curves; baryonic TullyFischer relation; mass discrepancy-acceleration relation.

\section{Contents}

\section{Introduction.}

2 Cosmological part. $\quad 2$

2.1 Universe mass as a function of cosmological Time. . . . . . . . . . . . . . . 2

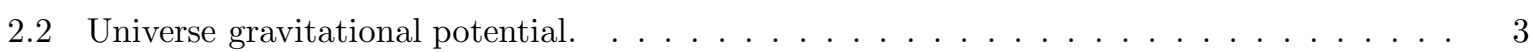

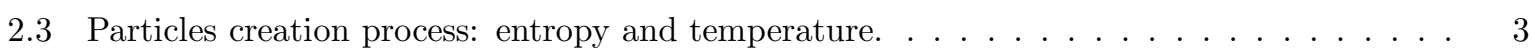

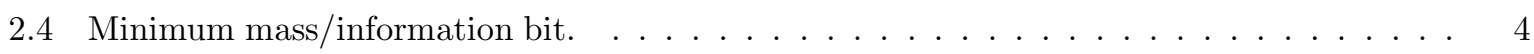

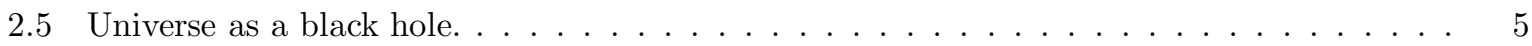

3 First law: the observed flattening of rotation curves in galaxies. $\quad 6$

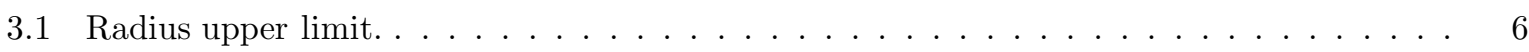


3.2 Radius lower limit. . . . . . . . . . . . . . . . . . . . . . . . 7

3.3 Asymptotic velocity limit. . . . . . . . . . . . . . . . . . . . . . 7

4 Second law: the baryonic Tully-Fisher relation. $\quad 8$

5 Third law: the mass discrepancy-acceleration relation. 9

\section{Introduction.}

According to Einstein's theory of general relativity spacetime has no intrinsic properties other than its curved geometry: it is merely a stage, albeit a dynamical one, on which matter moves under the influence of forces. There are well motivated reasons, coming from theory as well as observations, to challenge this conventional point of view. From the observational side, the fact that $95 \%$ of our Universe "consists of mysterious forms of energy or matter" gives sufficient motivation to reconsider this basic starting point. And from a theoretical perspective, insights from black hole physics and string theory indicate that our "macroscopic" notions of spacetime and gravity are emergent together from the entanglement structure of an underlying microscopic theory, requiring taking into account the entropy and temperature associated with the cosmological horizon [4].

\section{Cosmological part.}

Let's consider universe as the ensemble of $\aleph$ information bits or minimum particles of mass $m_{g}$ within volume $V$, with area $A$, radius $R$ and $\aleph \in \mathbb{Z}^{+}$. Thus, we can express universe mass $M$ as:

$$
M=\aleph \cdot m_{g}
$$

Thus, universe mass $M$ or universe energy $E_{U}=M c^{2}$ are divided evenly over the bits $\aleph$. The holographic principle states that the description of a volume of space can be thought of as $\aleph$ bits of information or minimum masses, encoded on a boundary to that region, a closed surface of area $A$. The information is distributed on the surface with each bit requiring an area equal to $l_{P}^{2}$, the so-called Planck area, from which $\aleph$ can thus be established as:

$$
\aleph=\frac{A}{l_{P}^{2}} \Rightarrow \aleph=\frac{c^{3} A}{\hbar G}
$$

Where $l_{P}=\sqrt{\frac{\hbar G}{c^{3}}}$ is the Planck length, $G$ is the universal gravitational constant, $c$ is the speed of light, and $\hbar$ is the reduced Planck constant. When substituted $\aleph$ in Eq.(1) we find:

$$
M=\frac{c^{3} A}{\hbar G} \cdot m_{g}
$$

\subsection{Universe mass as a function of cosmological Time.}

The age, cosmological Time or universe Time $t$ with regard to any relative reference system, is the elapsed time from the origin of the universe, according to the current theory of the Big Bang which may be considered until now as the most prudent and widely accepted model of the formation of the universe, to any Time $t$. Thus, if we include universe Time $t$ in Eq.(3), equation doesn't change and we find:

$$
M=\frac{c^{3} t}{G}\left(\frac{A m_{g}}{\hbar t}\right) \Rightarrow\left\{\begin{array}{l}
a) \quad M=\frac{c^{3}}{G} t \\
b) \quad A=\frac{\hbar}{m_{g}} t
\end{array}\right.
$$


Where we obtain universe mass at Time $t$ by option $a$ ) in Eq.(4), which is in perfect agreement to $M=\frac{c^{3}}{G H_{0}}$ according in [5] by approximation of $t_{0} \simeq \frac{1}{H_{0}}$, where $H_{0}$ is the Hubble constant at present Time $t_{0}$.

\subsection{Universe gravitational potential.}

If now, we include in Eq.(3) the universe radius $R$, equation doesn't change, and we can now find:

$$
\frac{G M}{R}=c^{2}\left(\frac{A m_{g} c}{\hbar R}\right) \Rightarrow \begin{cases}a) & \frac{G M}{R}=c^{2} \\ b) & A=\frac{\hbar}{m_{g} c} R\end{cases}
$$

Thus, the universe area $A$ can be expressed as the compton wavelength of minimum mass $m_{g}$ multiplied by universe radius $R$. Furthermore, if we now equate option b) of Eq.(5) and Eq.(4) respectively for universe area, we obtain universe radius at Time $t$ as:

$$
\frac{\hbar}{m_{g} c} R=\frac{\hbar}{m_{g}} t \Rightarrow R=c t
$$

However, according to Eq.(1) we can express any material particle or body of mass $m$ with $(m \in M)$ as: $m=n \cdot m_{g}$, where $\left(n \in \aleph ; n \in \mathbb{Z}^{+}\right)$. Therefore, by option $\left.a\right)$ of Eq.(5) we can now obtain the Universe gravitational Potential $\Phi$ as:

$$
m c^{2}+m \Phi=0 \quad \Longleftrightarrow \quad \Phi=-\frac{G M}{R}
$$

That is, there exists a non-local collective gravitational interaction of all particles within the Universe's Horizon, as a consequence of which all particles within the Universe are gravitationally entangled and form a unified statistical ensemble. Thus, we can relate the origin of inertia of any material particle to its interactions with the whole universe, according to the non-local potential of the whole universe, $\Phi$, acting on any material particle of the world ensemble as:

$$
m c^{2}=-m \Phi \quad \Longleftrightarrow \quad m c^{2}=\frac{G M m}{R} .
$$

Where $E=m c^{2}$ is the total energy of the particle. However, according to Eq.(7) and Eq.(6) for universe radius, we can obtain another consequence of non-local collective gravitational interaction of all particles within the universe's horizon due universe gravitational Potential $\Phi$, as:

$$
m \Phi=-m c^{2} \Rightarrow-\frac{G M m}{R^{2}}=m\left(-\frac{c^{2}}{R}\right) \quad \Rightarrow \quad-\frac{G M m}{R^{2}}=m\left(-\frac{c}{t}\right)
$$

That is, the existence of the Universe gravitational force $F_{G}=-\frac{G M m}{R^{2}}$ that causes a negative acceleration $a_{P}=-\frac{c}{t}$ on any material particle of the world ensemble.

\subsection{Particles creation process: entropy and temperature.}

We consider with regard to a relative reference system any universe Time interval $\Delta t=t-t_{i}$ according to option $a$ ) in Eq.(4). Thus, the universe mass must increase as Time progresses, that is, if the universe mass at some initial universe Time $t_{i}$ is $M\left(t_{i}\right)$ and the universe mass at any later universe Time $t$ is $M(t)$, then the increase of universe mass $\Delta M=M(t)-M\left(t_{i}\right)$ during elapsed proper time interval $\Delta t=t-t_{i}$ will be:

$$
\Delta M=\frac{c^{3}}{G} \Delta t \quad \Rightarrow \quad \Delta E_{U}=\Delta M c^{2}=\frac{c^{5}}{G} \Delta t \quad \Rightarrow \quad \Delta E_{U}=\frac{M c^{2}}{t} \Delta t
$$


Where $E_{U}=M c^{2}$ is the universe energy at Time $t$. However, by Eq.(1) we can now express Eq.(9) as:

$$
\Delta E_{U}=\left(\frac{\aleph}{t}\right) \cdot m_{g} c^{2} \Delta t
$$

Where $\left(\frac{\aleph}{t}\right)$ can be considered as the creation rate of minimum particles/bits. Therefore, universe increases its mass/energy as Time progresses by the creation of $\left(\frac{\aleph}{t}\right)$ new minimum particles of mass $m_{g}$ or information bits and energy $m_{g} c^{2}$ at every universe Time interval $\Delta t$. However as we know, according to the first law of thermodynamics, near the equilibrium any thermodynamic system at temperature $T$ experiences a state change according to equation:

$$
\Delta E=T \Delta S-\Delta W
$$

Where $\Delta E$ is the energy variation of the system, $T \Delta S$ is the work done by internal forces generating a change of entropy $\Delta S$ and $\Delta W$ is the quantity of energy lost by the system due to work done by the system on its surroundings. However, Universe can be considered a whole, there no surroundings out of it, thus $\Delta W=0$. Therefore, any universe state change is due only to internal forces and we can consider universe as a entropic system, that is, the entropy as a function of its internal energy. Applying now the first principle, the universe energy increase $\Delta E_{U}$ Eq.(9) can be expressed then as:

$$
\Delta E_{U}=T \Delta S \quad \Longleftrightarrow \quad \Delta=0
$$

Now, introducing the mass $m_{g}$ for the minimum particle or information bit, the reduced Planck's constant and the Boltzmann constant $k_{B}$ which relates the thermodynamic energy and temperature, equation doesn't change and we can now express the universe energy increase $\Delta E_{U}$ during any elapsed universe Time interval $\Delta t$ as:

$$
\Delta E_{U}=\frac{c^{5}}{G} \Delta t=T \Delta S \quad \Rightarrow \quad T \Delta S=\frac{\hbar c^{3}}{k_{B} G m_{g}} \cdot k_{B} \frac{m_{g} c^{2}}{\hbar} \Delta t
$$

And finally, we obtain by equalizing:

1. Temperature $T$ :

$$
T=\frac{\hbar c^{3}}{k_{B} G m_{g}}
$$

The temperature or thermal radiation $T$ in the horizon associated to the universe energy increase $\Delta E_{U}$ by the creation of $\left(\frac{\aleph}{t}\right)$ new minimum particles or information bits of mass $m_{g}$ and energy $m_{g} c^{2}$ during elapsed universe time interval $\Delta t$, Eq.(10).

2. Entropy increase $\Delta S$ :

$$
\Delta S=k_{B} \frac{m_{g} c^{2}}{\hbar} \Delta t
$$

The entropy increase on every minimum particle or bit of mass $m_{g}$ during elapsed universe time interval $\Delta t$.

\subsection{Minimum mass/information bit.}

Eq.(13) can also be expressed according to Eq.(1) and Eq.(4) by option $a$ ) for universe mass at Time $t$, as:

$$
T=\frac{\hbar c^{3}}{k_{B} G m_{g}} \Rightarrow T=\aleph \cdot \frac{\hbar c^{3}}{k_{B} G M} \Rightarrow T=\aleph \cdot T_{g} \Longleftrightarrow T_{g}=\frac{\hbar}{k_{B} t}
$$

Thus, Eq.(12) for universe energy increase $\Delta E_{U}$ during any elapsed universe Time interval $\Delta t$ can be now expressed as:

$$
T \Delta S=\left(\aleph \cdot \frac{\hbar}{k_{B} t}\right) \cdot k_{B} \frac{m_{g} c^{2}}{\hbar} \Delta t
$$


That is, temperature or thermal radiation $T$ in the horizon associated to the universe energy increase $\Delta E_{U}$ by the creation of new minimum particles or information bits equals to associate on every minimum particle $m_{g}$ a temperature: $T_{g}=\frac{\hbar}{k_{B} t}$.

However, by Eq.(1), the energy $E_{U}=M c^{2}$ is divided evenly over the bits $\aleph$, then temperature can be determined by the equipartition rule as the average energy per bit/minimum mass:

$$
M c^{2}=\frac{1}{2} \aleph k_{B} T_{g} \quad \Rightarrow \quad m_{g} c^{2}=\frac{1}{2} k_{B} T_{g}
$$

By simplifying previous equation according to Eq.(15), we find:

$$
m_{g} c^{2}=\frac{1}{2} \frac{\hbar}{t} \Rightarrow m_{g} c^{2}=\frac{1}{2} \hbar w \Longleftrightarrow w=\frac{1}{t}
$$

Where we obtain wave-particle duality as a function of universe Time. Furthermore, energy of minimum particle/bit $m_{g} c^{2}=\frac{1}{2} \hbar w$ is equivalent to Zero-point energy (ZPE), that is, the lowest possible energy that a quantum mechanical system may have, as expected, since $w=\frac{1}{t}$ as inverse of universe Time, can be considered as the minimum frequency. Therefore, Universe can be considered as a quantum mechanical system of $\aleph$ information bits or minimum particles of mass $m_{g}$.

However, the minimum energy $m_{g} c^{2}$ can be also expressed, according to the known relation $w=2 \pi \nu$ and $\hbar=\frac{h}{2 \pi}$, as:

$$
m_{g} c^{2}=\frac{1}{2} h \nu \Longleftrightarrow \nu=\frac{1}{t}
$$

Which can be approximated as:

$$
m_{g}=\frac{1}{2} \frac{h}{c^{2} t} \quad \Rightarrow \quad m_{g} \approx \frac{h H_{0}}{2 c^{2}}
$$

Thus, approaching $t_{0} \approx \frac{1}{H_{0}}$, the value $H_{0}=(74.03 \pm 1.42) \mathrm{kms}^{-1} \mathrm{Mpc}^{-1}$ in [6], we can obtain approximately: $m_{g} \sim 10^{-68} \mathrm{~kg}$ which can be in agreement with the range of values $m_{g} \sim\left(10^{-68}-10^{-66}\right) \mathrm{kg}$ for the entropic minimum mass found by the Prof. J. R. Mureika and R. B. Mann in [7] where this range represents the smallest non-zero mass for any particle quanta in the entropic gravity framework.

Now, according to Eq.(1), by option $a$ ) in Eq.(4) and Eq.(17), we can calculate the total number of minimum particles or bits of mass $m_{g}$ as:

$$
\aleph=\frac{2 c^{5} t^{2}}{\hbar G}
$$

Which, approaching $t_{0} \approx \frac{1}{H_{0}}$, we can obtain approximately: $\aleph \approx 10^{122}$.

\subsection{Universe as a black hole.}

The first indication of the emergent nature of spacetime and gravity comes from the laws of black hole thermodynamics [8]. As we know, a central role herein is played by the Bekenstein-Hawking entropy and Hawking temperature on the event horizon of a black hole given by:

$$
S_{B H}=k_{B} \frac{c^{3} A}{4 \hbar G} \quad \text { and } \quad T=\frac{\hbar c^{3}}{8 \pi G k_{B} M}
$$

Here $A$ denotes the area of the horizon, $\hbar$ is the reduced Planck constant, $c$ is the speed of light, $k_{B}$ is the Boltzmann constant, $G$ is the gravitational constant and $M$ is the mass of the black hole.

However, it's remarkable that Eq.(15) and Eq.(16) for universe energy increase $\Delta E_{U}$ during any elapsed universe Time interval $\Delta t$ can be now expressed as:

$$
T \Delta S=\frac{\aleph}{4} \cdot \frac{\hbar}{2 \pi k_{B} c}\left(\frac{8 \pi c}{t}\right) \cdot k_{B} \frac{m_{g} c^{2}}{\hbar} \Delta t
$$


Now, if we insert Eq.(2) for universe area as a function of total number of information bits/minimum masses, that is: $A=\aleph \cdot l_{p}^{2}$ in Bekenstein-Hawking entropy Eq.(20), we find:

$$
\frac{\aleph}{4}=\frac{S_{B H}}{k_{B}}
$$

Thus, $\frac{\aleph}{4}$ is equivalent to the a black hole entropy with area $A=\aleph \cdot l_{p}^{2}$ equal to the universe area. Therefore, Eq.(21) can be expressed now as:

$$
T \Delta S=\left[\left(\frac{S_{B H}}{k_{B}}\right) \cdot \frac{\hbar}{2 \pi k_{B} c}\left(\frac{8 \pi c}{t}\right)\right] \cdot k_{B} \frac{m_{g} c^{2}}{\hbar} \Delta t
$$

That is, it's as if the universe could be considered as a black hole emitting black body radiation where the temperature:

$$
T_{H U}=\frac{\hbar}{2 \pi k_{B} c}\left(\frac{8 \pi c}{t}\right)=\frac{\hbar a_{U}}{2 \pi k_{B} c}
$$

is the Hawking-Unruh temperature $T_{H U}$ and where $a_{U}=\frac{8 \pi c}{t}$ can be considered as the acceleration at the surface of the universe. This expansion acceleration $a_{U}$ at the surface will be considered in section $\S 4$ for baryonic Tully-Fisher relation.

\section{First law: the observed flattening of rotation curves in galaxies.}

The concept of "Dark Matter" was proposed by Prof. F. Zwicky to explain the anomalous rotation curves of the galaxies. The problem was that, according to Newtonian dynamics, the velocities of any body of mass $m$ at a distance $r$ from the center of the galaxy, must be expressed by assuming a circular orbit, as:

$$
\frac{m v^{2}}{r}-\frac{G M_{b} m}{r^{2}}=0 \quad \Rightarrow \quad v=\sqrt{\frac{G M_{b}}{r}}
$$

Where $M_{b}$ is the baryonic mass of the Galaxy, that is: the sum of its stars and gas: $M_{b}=M_{*}+M_{g}$, $m$ the mass of the body, $G$ the gravitational constant, $r$ the distance of the body to the center of the galaxy and $v$ its tangential velocity. However, the astronomical observations indicate that velocity of the rotation curves are flattened tending to a certain limit, instead of complying with the previous law where velocity must decrease as the radius $r$ increases.

\subsection{Radius upper limit.}

Now, we consider any gravitational local system (i.e., Galaxy) within the Universe of baryonic mass: $M_{b}=\aleph_{b} \cdot m_{g}$ according to Eq.(1) where $\left(M_{b} \in M\right)$ and $\left(\aleph_{b} \in \aleph\right)$.We will also consider that a material particle or body of mass $m$ is located at a distance $r$ from the center of the gravitational local system at Time $t$.

Now, we apply Newtonian Mechanics to our gravitational local system (Galaxy), this is: $\sum F_{i}=0$, then by Eq.(24) and according to Universe gravitational force Eq.(8) which acts on every particle or body of mass $m$ causing a negative acceleration $a_{P}=-\frac{c}{t}$, assuming a circular orbit, we obtain at Time $t$ the equation:

$$
\frac{m v^{2}}{r}-\frac{G M_{b} m}{r^{2}}-\frac{G M m}{R^{2}}=0 \quad \Rightarrow \quad v^{2}=\frac{G M_{b}}{r}+\frac{c r}{t}
$$


This equation we can now express it in two ways as follow and solving ${ }^{1}$ for $\left(\frac{G M_{b} t}{c r^{2}}\right)$, thus we obtain:

$$
\left.\begin{array}{l}
\text { 1) } v^{2}-\frac{2 c r}{t}=\frac{G M_{b}}{r}-\frac{c r}{t} \\
\text { 2) } \frac{c r}{v^{2} t}=\frac{1}{1+\frac{G M_{b} t}{c r^{2}}}
\end{array}\right\} \Rightarrow r^{2} \leq \frac{G M_{b} t}{c}
$$

Which can be considered as the radius upper limit of any gravitational system at Time $t$, as for example a rotating Galaxy, that is:

$$
r^{2} \leq \frac{G M_{b} t}{c} \Rightarrow r_{\infty}=\sqrt{\frac{G M_{b} t}{c}}
$$

\subsection{Radius lower limit.}

According to Eq.(25) and Eq.(26) we can obtain:

$$
v^{2}-\frac{2 G M_{b}}{r}=\frac{c r}{t}-\frac{G M_{b}}{r} \leq 0 \quad \Rightarrow \quad v^{2} \leq \frac{2 G M_{b}}{r}
$$

However, by Relativity any velocity $v$ is always less or equal to the constancy of the speed of light in the vacuum, that is, $v \leq c$. Thus, according to previous equation we obtain:

$$
\frac{2 G M_{b}}{r} \leq c^{2} \Longleftrightarrow v \leq c \quad \Rightarrow \quad r \geq \frac{2 G M_{b}}{c^{2}}
$$

Which establish a radius lower limit for a gravitational system at Time $t$ where we can verify that it corresponds to Schwarzschild radius.

\subsection{Asymptotic velocity limit.}

According to Eq.(25) we can express:

$$
\frac{c r^{2}}{t}=v^{2} r\left(1-\frac{G M_{b}}{v^{2} r}\right) \Rightarrow r=\frac{v^{2} t}{c}\left(1-\frac{G M_{b}}{v^{2} r}\right)
$$

Substituting this previous result for $r$ in Eq.(28) we have:

$$
v^{4} \leq \frac{2 G M_{b} c}{t}\left(\frac{1}{1-\frac{G M_{b}}{v^{2} r}}\right)
$$

However, by Eq.(28) we have: $\left(\frac{1}{2} \leq \frac{G M_{b}}{v^{2} r}\right) \Rightarrow\left(\frac{1}{2} \leq 1-\frac{G M_{b}}{v^{2} r}\right) \Rightarrow\left(\frac{1}{1-\frac{G M_{b}}{v^{2} r}}\right) \leq 2$. Therefore we obtain the velocity upper limit at Time $t$ as:

$$
v^{4} \leq \frac{4 G M_{b} c}{t} \Rightarrow v_{f}^{4}=\frac{4 G M_{b} c}{t}
$$

Thus, we prove that velocity upper limit or asymptotically flat rotation velocity $v_{f}$ depends on gravitational system baryonic mass and Universe Time $t$ and not on its radius. Thus, the flatness velocity curves are due to the gravitational force of whole Universe Eq.(8) acting on every material particle, which are gravitationally entangled. Therefore, we find for the first law [2]: rotation curves attain an approximately constant velocity, asymptotic or at rotation velocity $v_{f}$ according Eq.(30).

Approaching $t_{0} \approx \frac{1}{H_{0}}$, where $H_{0}$ is the Hubble constant at present Time $t_{0}$, we obtain for velocity upper limit: $v^{4} \leq 4 G M_{b} c H_{0}$. For example: for $H_{0}=(74.03 \pm 1.42) k m s^{-1} M p c^{-1}$ in [6] and the baryonic mass of our Milky Way $M_{b} \simeq 1.3 \times 10^{41} \mathrm{~kg}$ in [9], the rotational velocity limit will be: $v \leq 398 \mathrm{~km} / \mathrm{s}$, which establish a velocity upper limit for the astronomical observations.

\footnotetext{
${ }^{1}$ By simplicity we denote $B=\frac{c r}{v^{2} t}$ and $y=\frac{G}{c r_{b}} t$. Thus, we have by 2$)$ that: $B=\frac{1}{1+y} \Rightarrow B \leq 1, \forall y \geq 0$.

By 1) we have: $(1-2 B)=B(y-1) \quad \Rightarrow \quad(1-2 B) \leq(y-1) \Longleftrightarrow B \leq 1$. Now, by 2$)$ substituting value of $B$ in this equation and resolving for $y$, we obtain: $(y-1) \leq\left(y^{2}-1\right) \Rightarrow\left(y^{2} \geq y\right) \Rightarrow(y \geq 1) \quad \Rightarrow \quad \frac{G M_{b} t}{c} \geq r^{2}$
} 


\section{Second law: the baryonic Tully-Fisher relation.}

The Tully-Fisher Relation was published by astronomers R. Brent Tully y J. Richard Fisher in 1977 and was originally posed as an empirical relation between optical luminosity and the width of the $21 \mathrm{~cm}$ line.

The Baryonic Tully-Fisher Relation (BTFR) is currently an empirical relation between total baryonic mass and rotation velocity in disk galaxies [10], that is: $M_{b} \propto v^{4}$ and can be specifically expressed as:

$$
M_{b}=A v^{x}
$$

It's suffices to describe the data for rotating galaxies. There is no indication of any need for a third parameter such as radius or surface brightness. Astronomical observations data for galaxies strongly suggest that Baryonic Tully-Fisher Relation has the form:

$$
M_{b}=A v^{4}
$$

where $A=\left(a_{0} G\right)^{-1}$ with $a_{0} \cong 1.2 \times 10^{-10} \mathrm{~ms}^{-2}$ from astronomical observations and $H_{0}$ is the Hubble constant at current universe Time $t_{0}$ and where $a_{0} \cong \frac{c H_{0}}{2 \pi}$ according [11]. The value of $a_{0}$ appears universal and perhaps can be a quantum gravity effect [12].

Now, according to Eq.(25) we can also express the rotational velocity $v$ at Time $t$ by two different ways as:

$$
v^{2}=\frac{G M_{b}}{r}\left(1+\frac{c r^{2}}{G M_{b} t}\right) \quad \vee \quad v^{2}=\frac{c r}{t}\left(1+\frac{G M_{b} t}{c r^{2}}\right)
$$

Thus, by multiplying these two equations, the fourth power of the rotational velocity of the galaxy can be expressed at Universe Time $t$, as:

$$
v^{4}=a(t) G M_{b} \quad \Longleftrightarrow \quad a(t)=\frac{c}{t}\left(1+\frac{c r^{2}}{G M_{b} t}\right)\left(1+\frac{G M_{b} t}{c r^{2}}\right)
$$

If we develop for $a(t)$ we obtain:

$$
a(t)=\frac{c}{t}\left(2+\frac{c r^{2}}{G M_{b} t}+\frac{G M_{b} t}{c r^{2}}\right) \Rightarrow a(t) \leq \frac{4 c}{t}
$$

Since by Eq.(26): $\frac{c r^{2}}{G M_{b} t} \leq 1$, it is easily proved that: $\frac{c r^{2}}{G M_{b} t}+\frac{G M_{b} t}{c r^{2}} \leq 2$. Therefore, according to previous equation we obtain: $a(t) \in\left(\frac{2 c}{t}, \frac{4 c}{t}\right]$ and we can now express Eq.(33) as:

$$
M_{b}=A v^{4} \Longleftrightarrow A=[a(t) G]^{-1}
$$

Since $a(t)$ can be considered as approximately constant in this short interval $\left(\frac{2 c}{t}, \frac{4 c}{t}\right]$, which could explain why Eq.(31) remains valid for $x=4$ with $A \equiv$ constant. However, according to Hubble law we can express the Hubble parameter $H(t)$, which is related with universe Time $t$, as:

$$
H(t)=\frac{a_{U}}{v_{U}}
$$

Where $a_{U}$ and $v_{U}$ can be considered as the universe acceleration and velocity respectively. If now, we insert $a_{U}=\frac{8 \pi c}{t}$ as the expansion acceleration at the surface of the universe according to Eq.(22) and Eq.(23) that can be considered as the universe acceleration, then for any $v_{U} \leq c$ according to relativity, we obtain:

$$
H(t)=\frac{8 \pi c}{v_{U} t} \Rightarrow H(t) \cdot t=8 \pi\left(\frac{c}{v_{U}}\right) \quad \Rightarrow \quad H(t) \cdot t \geq 8 \pi
$$

Therefore by substituting this value into the Eq.(33) and Eq.(34) we finally find:

$$
v^{4} \leq\left(\frac{c H(t)}{2 \pi}\right) G M_{b} \quad \Rightarrow \quad v_{f}^{4}=a_{0} G M_{b} \Longleftrightarrow a_{0}=\frac{c H_{0}}{2 \pi}
$$

Where $H_{0}=H\left(t_{0}\right)$ is the Hubble constant at present Time $t_{0}$ and thus: $M_{b}=A v_{f}^{4} \Longleftrightarrow A=\left[a_{0} G\right]^{-1}$ according to [10]. Thus, we find for the second law: the observed baryonic mass scales is the fourth power of the amplitude of the flat rotation $v_{f}$. 


\section{Third law: the mass discrepancy-acceleration relation.}

The mass discrepancy-acceleration relation proposed in ([2][3]) is considered as the third law of galactic rotation, that is, there is a relation between the squared ratio of the observed velocity $V$ to that predicted by the observed baryons $V_{b}$ and the observed distribution of baryonic matter, thus:

$$
\frac{V^{2}}{V_{b}^{2}} \cong \frac{M_{t o t}}{M_{b}}
$$

where $\frac{M_{\text {tot }}}{M_{b}}$ is defined by prof. McGaugh as the "discrepancy mass". The centripetal acceleration predicted by Newtonian gravity Eq.(24) for the observed baryonic mass components [2], is:

$$
g_{N}(r)=\frac{V_{b}^{2}}{r}=\frac{G M_{b}}{r^{2}}
$$

and the observed centripetal acceleration produced by all mass components:

$$
g_{o b s}(r)=\frac{V^{2}}{r}
$$

Therefore, there is a one-to-one correspondence between the radial force and the observed distribution of baryonic matter, that is: the mass discrepancy-acceleration relation:

$$
\frac{g_{o b s}(r)}{g_{N}(r)}=\frac{V^{2}}{V_{b}^{2}} \cong \frac{M_{t o t}}{M_{b}}
$$

However, Eq.(25) can be expressed as:

$$
v^{2}=\frac{G M_{b}}{r}\left(1+\frac{c r^{2}}{G M_{b} t}\right) \Rightarrow v^{2}=\frac{G M_{b}}{r}\left(1+\frac{M_{D}}{M_{b}}\right) \Longleftrightarrow M_{D}=\frac{c r^{2}}{G t}
$$

where the quantity $M_{D}$ has mass dimensions and therefore "the missing mass" is not missing, it's due to the universe gravitational potential acting on every material particle, which are gravitationally entangled. This Eq.(37) can be also now expressed as:

$$
v^{2}=\frac{G M_{b}}{r}\left(1+\frac{M_{D}}{M_{b}}\right) \Rightarrow\left\{\begin{array}{l}
a) \quad v^{2}=\frac{G M_{b}}{r}+\frac{G M_{D}}{r}=V_{b}^{2}+V_{D}^{2} \\
b) \quad v^{2}=\frac{G}{r}\left(M_{b}+M_{D}\right)=\frac{G M_{t o t}}{r}
\end{array}\right.
$$

with $M_{t o t}$ as the sum of all mass components:

$$
M_{t o t}=M_{b}+M_{D} \quad \Rightarrow \quad M_{t o t}=M_{*}+M_{g}+M_{D}
$$

with baryonic mass $M_{b}=M_{*}+M_{g}$ as the sum of its stars and gas. The Eq.(38) for option $a$ ) is the same one found by prof. McGaugh in [2] with $V_{D}^{2}$ as the supposed circular velocity due to the "dark matter halo". Thus, we find:

$$
V_{D}^{2}=\frac{G M_{D}}{r} \Rightarrow V_{D}^{2}=\frac{c r}{t} \Longleftrightarrow M_{D}=\frac{c r^{2}}{G t}
$$

Therefore, we can now express Eq.(37) or Eq.(38) as:

$$
v^{2}=V_{b}^{2}\left(1+\frac{M_{D}}{M_{b}}\right) \Rightarrow \frac{v^{2}}{V_{b}^{2}}=\frac{M_{b}+M_{D}}{M_{b}} \Rightarrow \frac{v^{2}}{V_{b}^{2}}=\frac{M_{t o t}}{M_{b}}
$$

which is the mass discrepancy-acceleration relation proposed by prof. McGaugh according to Eq.(35) and Eq.(36). 


\section{References}

[1] Dimensional reduction in Quantum Gravity

Gerard 't Hooft.

arXiv:gr-qc/9310026 (1993).

[2] The Third Law of Galactic Rotation.

Stacy S. McGaugh.

Galaxies 2014, 2(4), 601-622; https://doi.org/10.3390/galaxies2040601.

[3] The Mass Discrepancy-Acceleration Relation: Disk Mass and the Dark Matter Distribution.

Stacy S. McGaugh.

arXiv:astro-ph/0403610v4 29 Sep 2004.

[4] Emergent Gravity and the Dark Universe,

Erik Verlinde.

arXiv:1611.02269v2 [hep-th]; SciPost Phys. 2, 016 (2017).

[5] Derivation of the mass of the observable universe.

Carvalho, J.C.

Int J Theor Phys 34, 25072509 (1995). https://doi.org/10.1007/BF00670782

[6] Large Magellanic Cloud Cepheid Standards Provide a 1\% Foundation for the Determination of the Hubble Constant and Stronger Evidence for Physics Beyond $\Lambda$ CDM.

Riess, Adam G.; Casertano, Stefano; Yuan, Wenlong; Macri, Lucas M.; Scolnic, Dan .

The Astrophysical Journal, 876:85 (13pp), 2019 May 1. doi:10.3847/1538-4357/ab1422

arXiv:1903.07603v2 [astro-ph.CO].

[7] Does Entropic Gravity Bound the Masses of the Photon and Graviton?.

J. R. Mureika and R. B. Mann.

arXiv:1005.2214v1 [gr-qc].

[8] Four laws of black hole mechanics.

J. M. Bardeen, B. Carter and S. W. Hawking.

Commun. math. Phys. 31, 161-170 (1973).

[9] M. Boylan-Kolchin et al.

The Astrophysical Journal 768, 140 (2013)

[10] The baryonic Tully-Fischer relation of gas rich Galaxies as a test of $\Lambda$ CDM and MOND,

Stacy S. McGaugh.

arXiv:1107.2934v2.

[11] MOND theory.

Mordehai Milgrom.

arXiv:1404.7661v2 [astro-ph.CO].

[12] MOND as a regime of quantum gravity,

Lee Smolin.

arXiv:1704.00780v2. 